\title{
Retrait de consentement et chirurgie éveillée : illustration et enjeux éthiques
}

\author{
Michel Wager, Foucaud Du Boisgueheneuc, Coline Bouyer, Claudette Pluchon, \\ Véronique Stal, Roger Gil
}

\begin{abstract}
RÉSUMÉ: Contexte: Le développement au cours des deniers années des interventions neurochirurgicales sous anesthésie locale, en particulier dans le champ neuro-oncologique, rend possible la question d'une éventuelle révocation per opératoire du consentement du patient à la procédure chirurgicale. Observation: Les auteurs décrivent la révocation de son consentement par un patient au cours de l'exérèse chirurgicale d'un gliome de bas grade fronto-temporo-insulaire droit. Discussion: Les aspects spécifiques aux conditions de révocation du consentement dans le contexte particulier de la chirurgie sous anesthésie locale sont discutés. La possibilité d'un pacte d'Ulysse est proposée et discutée. Conclusions: Les interventions chirurgicales en condition éveillée créent un contexte particulier en ce qu'une révocation du consentement per opératoire devient possible. Dans ces conditions, l'information préopératoire pourrait aussi aborder avec le malade la question de la conduite à tenir au cas où il serait amené à solliciter un retrait de consentement lors de son réveil per opératoire.
\end{abstract}

doi:10.1017/cjn.2014.26

Can J Neurol Sci. 2014; 41: 590-596

\section{Observation Clinque}

Un patient né le 0102 1990, maçon en recherche d'emploi et célibataire vivant chez ses parents, sans antécédent notable, droitier homogène, présente en avril 2012 un état de mal épileptique sur hypertension intracrânienne révélant un gliome de bas grade fronto-temporo-insulaire droit avec expansion frontale gauche visible sur l'IRM cérébrale initiale du 30 mai 2012 (Figure 1-a). L'examen neuropsychologique préopératoire réalisé le 9 mai 2012 montre une efficience cognitive globale satisfaisante avec, à l'ERFC (1), un score de 48 pour un maximum de 50 et un seuil pathologique à 46. La batterie rapide d'évaluation frontale (2) montre un discret syndrome dysexécutif. Il existe un déficit de la mémoire de travail, explorée par le subtest de Mémoire de chiffres de la WAIS, tandis que les scores obtenus au test de Stroop et au Trail Making restent dans les limites de la normale (3). Le score obtenu à l'échelle d'apathie de Lille est normal (4). Il manifeste une légère anxiété sans dépression (5). Après discussion en réunion de concertation pluridisciplinaire de neuro-oncologie, une résection tumorale en condition éveillée est proposée au patient afin de réaliser des tests peropératoires permettant de guider l'acte chirurgical et ainsi d'obtenir le meilleur rapport risques-bénéfices du point de vue onco-fonctionnel. Cette intervention est réalisée le 8 juin 2012, selon la séquence « Endormi / Eveillé / Endormi » adoptée dans notre institution. Ainsi, après réalisation de la voie d'abord chirurgicale sous anesthésie générale, le patient est réveillé sans difficulté particulière. On réalise l'exérèse de la portion la plus antérieure, soit la portion frontale droite de la tumeur, en faisant exécuter une tâche motrice et en progressant d'avant en arrière. Mais le patient se déclare assez rapidement très fatigué, ne s'oppose pas aux tests, mais s'avère incapable de toute mobilisation attentionnelle. Il est alors décidé de ne pas réséquer les portions insulaire droite et frontale gauche de la tumeur. En effet, il apparait à ce stade de l'intervention que la coopération du patient sera insuffisante pour lui permettre de réaliser les taches motrices (limite de résection interne de la portion insulaire) et de programmation (résection frontale controlatérale) nécessaires. Il est donc décidé de rendormir le patient, et de proposer la réalisation d'une exérèse en deux temps, en réalisant ultérieurement une seconde intervention en condition éveillée.

Les suites opératoires de cette première intervention sont sans particularité.

Le bilan neuropsychologique réalisé le 26 septembre 2012 (Table 1) montre une efficience cognitive globale satisfaisante avec, à l'ERFC, un score de 49 pour un maximum de 50 et un seuil pathologique à 46. Il n'existe pas de signe de détérioration psychométrique par rapport à l'efficience estimée antérieurement. En ce qui concerne les fonctions exécutives, la batterie rapide d'évaluation frontale montre un score satisfaisant, donc amélioré par rapport à l'évaluation qui avait précédé la première intervention. Cependant la fluence verbale littérale est diminuée alors que la fluence catégorielle est satisfaisante. Le Trail Making test ne montre pas de déficit de la flexibilité mentale. Il existe à l'échelle HADS une légère élévation du score d'anxiété, alors qu'il n'apparait aucune manifestation dépressive. La Lille Apathy Rating Scale indique une apathie modérée. Il existe une dysprosodie émotionnelle expressive alors que la reconnaissance de la prosodie émotionnelle est normale. Il existe des difficultés pour reconnaitre au test d'Ekman $(6,7)$ certaines émotions faciales comme le dégoût et la surprise. Il n'y a pas d'alexithymie (échelle de Toronto, TAS 20 (8)).

From the Service de Neurochirurgie (MW), Service de Neurologie (FDB, CB, CP), Service de Neurophysiologie Clinique (VS), Centre Hospitalier Universitaire de Poitiers, France; Université de Poitiers (RG), Responsable de l'Espace Ethique de la Région Poitou-Charentes, Centre Hospitalier Universitaire de Poitiers, France.

Received March 17, 2014. Final Revisions Submitted April 2, 2014.

Correspondence to: Michel Wager, Service de Neurochirurgie, Centre Hospitalier

Universitaire de Poitiers, 2 Rue de La Milétrie, BP 577, 86021 Poitiers Cedex, France.

Email: m.wager@chu-poitiers.fr 
(a)

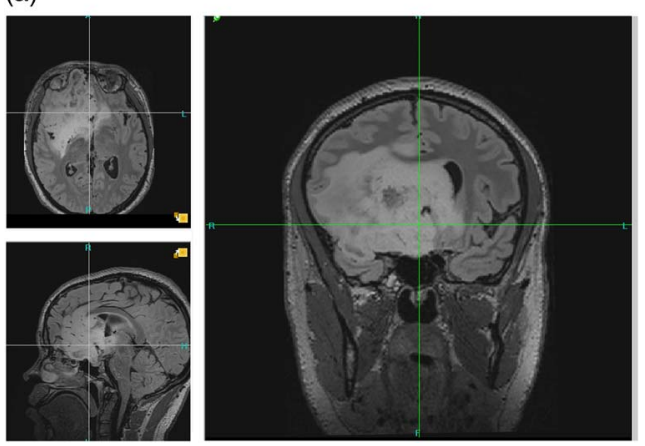

Figure 1a: IRM du 30 mai 2012. Avant la première intervention Séquences axiale, sagittale et coronale FLAIR. On n'observait pas de rehaussement sur les séquences $T 1$ après injection de gadolinium.

(b)

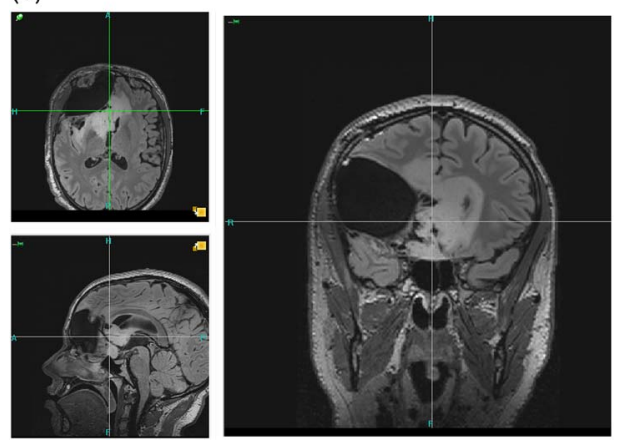

Figure 1b: IRM du 25 septembre 2012. Préopératoire de la seconde intervention

Séquences axiale, sagittale et coronale FLAIR. On n'observait pas sur la séquence $T 1$ de rehaussement après injection de gadolinium.

(c)

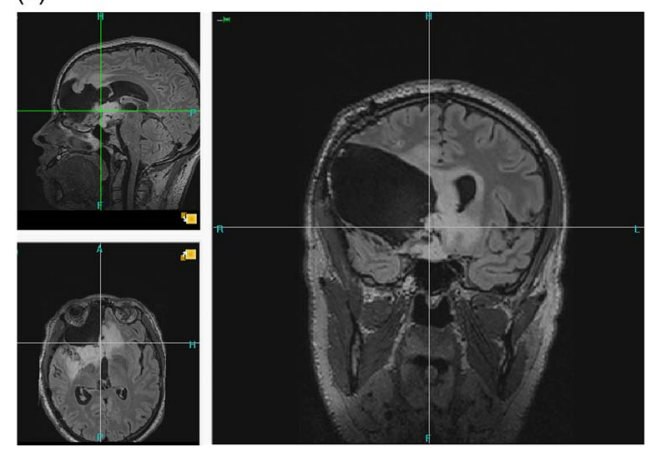

Figure 1c: IRM du 22 janvier 2013. Après la seconde intervention Séquences axiale, sagittale et coronale FLAIR. On n'observait pas de rehaussement sur les séquences T1 après injection de gadolinium.

Le tableau 1 résume les principaux résultats des tests pour lesquels le patient avait obtenu un score satisfaisant, donc amélioré par rapport à l'évaluation qui avait précédé la première intervention. Le malade, demandeur d'emploi, n'a pas de travail et la qualité de son insertion professionnelle ne peut être évaluée mais il déclare mener une vie familiale normale. Il se plaint seulement d'un ralentissement (Table 1).
L'examen anatomopathologique a conclu à un gliome diffus de bas grade de l'adulte, de phénotype oligodendroglial, de grade II (9). Une IRM au décours de cette première intervention a permis de vérifier le caractère très partiel de cette première résection (IRM du 25 septembre 2012 - Figure 1-b), et compte tenu de l'enjeu oncologique, une seconde intervention est programmée, de nouveau en condition éveillée, afin de compléter la première exérèse. Cette intervention a lieu quatre mois après la première, au mois d'octobre 2012.

Le patient est de nouveau informé du but de l'intervention, qui est de permettre une exérèse aussi large que possible du reliquat tumoral en limitant les risques fonctionnels par la réalisation de tests préopératoires nécessitant un réveil. Le patient donne son accord. En outre, compte tenu des difficultés de réveil lors de la première opération, il est décidé de réaliser cette seconde intervention sous anesthésie locale avec une sédation particulièrement allégée.

C'est en effet sous sédation que sont réalisés l'incision cutanée, le décollement du muscle temporal et le soulèvement du volet osseux: la voie d'abord chirurgicale est identique à celle de la première opération. La sédation est interrompue et le réveil est de très bonne qualité. La stimulation du cortex moteur à un seuil de 2,5 milliampères déclenche lors de la stimulation de l'opercule frontal une crise comitiale partielle brève. La résection tumorale débute mais le patient se plaint alors d'une grande soif, plainte qu'il réitère plusieurs fois de suite en signalant aussi une intense fatigue. Non seulement le patient s'avère indisponible pour les tests préopératoires, mais il demande clairement d'en rester là, d'interrompre l'intervention en condition éveillée et d'être ré-endormi. Le patient réitérant la même demande à plusieurs reprises, il est informé qu'une fois ré-endormi l'on procèdera à l'exérèse de ce qui peut l'être sans le contrôle permis par l'éveil. Il accepte cette démarche et il est alors replacé sous anesthésie générale (Table 2 - Verbatim, et Film Opératoire - Video). Il n’est alors réalisé qu'une exérèse partielle du reliquat tumoral au niveau du corps calleux. La ligne médiane ne sera pas franchie dans le souci de préserver les fonctions exécutives. Le reliquat insulaire est laissé en place puisqu'il s'avère impossible de garantir l'intégrité des voies longues médialement à l'insula. De la même manière l'impossibilité de tout contrôle des fonctions frontales de l'hémisphère majeur conduit à laisser en place le reliquat tumoral frontal gauche.

Les suites opératoires sont simples. Un bilan postopératoire à trois mois est réalisé en janvier 2013. L'IRM du 22 janvier 2013 ne montre pas, à ce délai court, d'évolutivité manifeste de la tumeur Figure 1-c. Le malade présente une apathie modérée, un peu plus marquée qu'en préopératoire. Les tests neuropsychologiques explorant les fonctions exécutives restent dans la norme sauf la fluence littérale qui reste déficitaire mais non aggravée. Il apparaît une légère apathie. Le score d'anxiété s'est normalisé. La Table 1 montre l'évolution du bilan neuropsychologique à quatre mois de la seconde intervention (Table 1).

Le suivi ultérieur a montré une progression remnologique du reliquat tumoral, et une chimiothérapie par témozolomide a été débutée au mois de mai 2013. Lors de la consultation du mois de novembre 2013, le patient va bien et mène la même vie qu'avant son hospitalisation initiale. Il déclare ne conserver aucun mauvais souvenir des deux interventions, et en particulier de la seconde. Interrogé sur son retrait de consentement, il dit seulement qu'il n'a à se plaindre de rien et que tout s'est passé comme il l'avait souhaité. 
Table 1: Résultats pré et post opératoires à la batterie de tests explorant les fonctions cognitives globales et les fonctions exécutives et aux échelles évaluant le statut psycho-comportemental.

\begin{tabular}{|c|c|c|c|c|c|c|c|}
\hline & & \multicolumn{2}{|c|}{$09 / 05 / 2012$} & \multicolumn{2}{|c|}{$26 / 09 / 2012$} & \multicolumn{2}{|c|}{$21 / 01 / 2013$} \\
\hline \multicolumn{2}{|c|}{ Test d'Evaluation Rapide des fonctions Cognitives } & \multicolumn{2}{|c|}{$48 / 50(\mathrm{NP})$} & \multicolumn{2}{|c|}{$49 / 50(\mathrm{NP})$} & \multicolumn{2}{|c|}{$48,5 / 50(\mathrm{NP})$} \\
\hline \multicolumn{2}{|l|}{ Batterie Rapide d'Evaluation Frontale } & \multicolumn{2}{|r|}{$13 / 18(\mathrm{P})$} & \multicolumn{2}{|c|}{$16 / 18(\mathrm{NP})$} & \multicolumn{2}{|c|}{$16 / 18(\mathrm{NP})$} \\
\hline \multirow[t]{2}{*}{ Wisconsin Card Sorting Test } & Séries & \multicolumn{2}{|r|}{$6(\mathrm{NP})$} & \multicolumn{2}{|r|}{$5(\mathrm{NP})$} & \multicolumn{2}{|r|}{$6(\mathrm{NP})$} \\
\hline & $\%$ erreurs pathologiques & \multicolumn{2}{|r|}{$0 \%(\mathrm{NP})$} & \multicolumn{2}{|r|}{$38 \%(\mathrm{NP})$} & \multicolumn{2}{|r|}{$0 \%(\mathrm{NP})$} \\
\hline Stroop Test & SR-SP & $\begin{array}{l}-4,79 \\
(\mathrm{NP})\end{array}$ & $\begin{array}{c}\mathrm{z} \text {-score }=-0,47 \\
(\mathrm{NP})\end{array}$ & $\begin{array}{c}0,2 \\
(\mathrm{NP})\end{array}$ & $\begin{array}{c}\text { z-score }=+0,02 \\
(\mathrm{NP})\end{array}$ & $\begin{array}{l}10,8 \\
(\mathrm{NP})\end{array}$ & $\begin{array}{c}\text { z-score }=+1,08 \\
(\mathrm{NP})\end{array}$ \\
\hline Trail Making Test & B-A & $\begin{array}{l}39^{\prime \prime} \\
(\mathrm{NP})\end{array}$ & $\begin{array}{c}\text { z-score }=+0,12 \\
(\mathrm{NP})\end{array}$ & $15^{\prime \prime}$ & $\begin{array}{c}\text { z-score }=+0,84 \\
(\mathrm{NP})\end{array}$ & $33^{\prime \prime}$ & $\begin{array}{c}\text { z-score }=+0,30 \\
(\mathrm{NP})\end{array}$ \\
\hline Fluence catégorielle (catégorie Animaux) & Mots fournis en 1c & 17 & z-score $=-1,05$ & 13 & z-score $=-1,66$ & 13 & z-score $=-1,8$ \\
\hline Fluence littérale (lettre M) & Mots fournis en 1 & 7 & z-score $=-1,77$ & 5 & z-score $=-2,18$ & 5 & $\mathrm{z}$-score $=-2,18$ \\
\hline Digit Span & $\begin{array}{c}\text { Note standard } \\
\text { (WAIS III) }\end{array}$ & $\begin{array}{c}3 / 19 \\
(\mathrm{P})\end{array}$ & $\begin{array}{c}\text { z-score }=-2,33 \\
(P)\end{array}$ & $\begin{array}{l}7 / 19 \\
(\mathrm{NP})\end{array}$ & $\begin{array}{c}\text { z-score }=-1 \\
(\mathrm{NP})\end{array}$ & $\begin{array}{l}8 / 19 \\
(\mathrm{NP})\end{array}$ & $\begin{array}{c}\text { z-score }=-0,66 \\
\text { (NP) }\end{array}$ \\
\hline \multirow[t]{8}{*}{ Ekman's pictures of facial affects } & Joie & \multicolumn{2}{|r|}{$7 / 7$} & \multicolumn{2}{|r|}{$7 / 7$} & \multicolumn{2}{|r|}{$7 / 7$} \\
\hline & Tristesse & \multicolumn{2}{|r|}{$2 / 7$} & \multicolumn{2}{|r|}{$4 / 7$} & \multicolumn{2}{|r|}{$2 / 7$} \\
\hline & Dégoût & \multicolumn{2}{|r|}{$4 / 7$} & \multicolumn{2}{|r|}{$1 / 7$} & \multicolumn{2}{|r|}{$3 / 7$} \\
\hline & Colère & \multicolumn{2}{|r|}{$6 / 7$} & \multicolumn{2}{|r|}{$5 / 7$} & \multicolumn{2}{|r|}{$7 / 7$} \\
\hline & Peur & \multicolumn{2}{|r|}{$6 / 7$} & \multicolumn{2}{|r|}{$7 / 7$} & \multicolumn{2}{|r|}{$7 / 7$} \\
\hline & Surprise & & $6 / 7$ & & $1 / 7$ & & $3 / 7$ \\
\hline & Neutre & & $6 / 7$ & & $6 / 7$ & & $6 / 7$ \\
\hline & Total & & $37 / 49$ & & $31 / 49$ & & $35 / 49$ \\
\hline Echelle d'Alexithymie de Toronto/TAS-20 & Score global & & 39 (NP) & & 45 (NP) & & $39(\mathrm{NP})$ \\
\hline Hospital Anxiety and Depression Scale & Anxiété & $9 / 21$ & nxiété probable) & $10 / 2$ & (anxiété certaine) & & 6/21 (NP) \\
\hline & Dépression & & 2/21 (NP) & & $4 / 21(\mathrm{NP})$ & & $3 / 21(\mathrm{NP})$ \\
\hline Lille Apathy Rating Scale & & & $\begin{array}{l}-25 / 36 \\
(\mathrm{NP})\end{array}$ & & $\begin{array}{l}-18 / 36 \\
\text { nnce à l'apathie) }\end{array}$ & & $\begin{array}{l}-11 / 36 \\
\text { athie modérée) }\end{array}$ \\
\hline
\end{tabular}

$(P=$ pathologique; $N P=$ non pathologique; $S R-S P=$ score réel-score prédictif (Stroop test) $; B-A=$ temps Partie B-temps Partie A (Trail Making test). Les résultats ont été considérés comme pathologiques à partir d'un z-score égal ou inférieur à -2)

\section{DISCUSSION}

Une révocation de consentement a pu être observée au cours d'une chirurgie éveillée pour implantation d'électrodes de stimulation cérébrale profonde (10). Le cas rapporté ici ne concerne pas un acte de chirurgie fonctionnelle mais un acte de chirurgie éveillée pour exérèse d'un gliome. La question posée est de savoir en quoi la révocation d'un consentement au cours d'un éveil chirurgical diffère de la révocation d'un consentement au cours des autres actes chirurgicaux classiquement effectués sous anesthésie générale jusqu'à la fin de l'intervention et au cours des autres procédures médicales de soins ou de recherche clinique.

\section{Le retrait du consentement est un droit fondamental du malade}

De manière générale, le consentement donné par un malade pour subir un examen complémentaire ou pour être enrôlé dans un protocole de recherche clinique peut être retiré à tout moment. Ainsi, par exemple, le Protocole additionnel à la Convention européenne sur les Droits de l'Homme et la biomédecine, relatif à la recherche biomédicale (11) stipule dans son article 14: «Aucune recherche sur une personne ne peut être effectuée... sans que cette personne ait donné son consentement éclairé, libre, exprès, spécifique et consigné par écrit. Ce consentement peut être librement retiré par la personne à tout moment de la recherche ». Le recueil d'un consentement est un devoir du médecin mais le consentement donné par le malade n'est jamais pour lui un engagement: le malade peut révoquer son consentement à tout moment sans avoir à donner de justifications, que ce soit avant la réalisation de l'acte médical ou pendant sa réalisation. Ainsi un malade peut demander par exemple à ce qu'une IRM soit interrompue; un autre peut décider de ne pas poursuivre l'essai d'un nouveau médicament pour lequel il avait d'abord consenti. Le principe d'autonomie interdit de considérer qu'un malade est lié par son consentement. Ainsi les recommandations formulées en 2007 par le comité international de bioéthique (UNESCO) précisent que: « Le consentement est l'un des principes de base de la bioéthique parce qu'il est étroitement lié au principe d'autonomie et parce qu'il reflète l'affirmation des droits de l'homme et de la dignité humaine, qui sont des valeurs fondamentales des sociétés démocratiques... Il faut également souligner que le consentement à une intervention médicale ou scientifique particulière implique le droit de retirer librement le consentement à tout moment » (12). Il existe donc en bioéthique un accord international pour souligner que le recueil du consentement 


\section{Table 2: Verbatim de la conversation: « Retrait de Con-} sentement Per Opératoire».

\begin{tabular}{l}
\hline Durée complète de la discussion: une minute. \\
\hline $\begin{array}{l}\text { Ce qui vous empêche de continuer, c'est parce que vous avez mal quelque part, ou } \\
\text { parce que vous êtes mal installé? }\end{array}$ \\
\hline C'est parce que je ne me sens pas capable de continuer. Je suis fatigué. J'ai soif. \\
\hline $\begin{array}{l}\text { D'accord. Donc, c'est à la fois la fatigue et puis la soif qui vous empêchent de } \\
\text { continuer? C'est ça? }\end{array}$ \\
\hline Oui, voilà, oui. \\
\hline Mais ce n'est pas la douleur? \\
\hline Non, c'est que je suis incapable de continuer. Je suis fatigué. \\
\hline Bon. Ce que l'on va faire, c'est que l'on va enlever ce que l'on pourra (de la tumeur), en \\
sachant que vous êtes endormi. \\
\hline Oui. Excusez-moi, Monsieur Wager, mais je suis incapable de continuer. Je ne peux \\
pas continuer. \\
\hline Vous vous sentez trop fatigué? D'accord. On va vous rendormir, dans ce cas. \\
\hline Oui, s'il vous plait, oui. \\
\hline Oui, l'on va vous rendormir. \\
\hline Je voudrais bien rentrer dans ma chambre, car je suis vraiment fatigué. \\
\hline Oui, je comprends ça. \\
\hline Fin de l'enregistrement. \\
\hline
\end{tabular}

est coextensif au respect du principe d'autonomie et de la dignité de la personne humaine et que tout consentement d'une personne implique le droit de retirer ce consentement à tout moment. La personne qui consent ne peut jamais être liée par son consentement.

\section{Le consentement doit être libre, exprès et éclairé}

La validité d'un consentement nécessite certaines précautions. Le consentement doit être libre, c'est-à-dire sans pression. Un médecin ne peut pas forcer le malade à consentir en le menaçant de suspendre sa prise en charge au cas où le malade refuserait un examen ou un traitement. Le consentement doit être exprès ce qui veut dire explicite et donc clairement manifesté. La signature d'un formulaire de consentement, même si elle est souvent demandée n'est jamais la preuve formelle du caractère explicite d'un consentement. C'est d'ailleurs pour tenter de prouver le caractère explicite d'un consentement que certains formulaires demandent à ce que la signature soit précédée de la mention «Lu et approuvé ». Car le consentement, pour être explicite, doit être précédé d'une information. Cette information doit être claire, c'est-à-dire donnée sans jargon technique et en s'assurant que le malade comprend ce qui lui est dit. La Déclaration de Lisbonne de l'Association Médicale Mondiale sur les Droits du Patient (13) précise par exemple que: tout patient « a droit à l'information nécessaire pour prendre ses décisions. Il doit pouvoir clairement comprendre l'objet d'un examen ou d'un traitement, les effets de leurs résultats et les conséquences d'un refus de consentement ».

\section{Le consentement aux actes chirurgicaux sous anesthésie}

Le consentement donné à un acte chirurgical effectué sous anesthésie a cette particularité qu'il ne peut pas être retiré pendant l'acte chirurgical lui-même puisque le malade, une fois endormi, est dépourvu de toute capacité d'exercer son autonomie. Certes le chirurgien au moment où il a recueilli le consentement doit avoir donné tous les renseignements nécessaires non seulement sur l'acte chirurgical lui-même mais aussi sur les complications qui pourraient survenir et les modifications que ces complications pourraient entraîner sur la réalisation de l'acte chirurgical lui-même. Mais s'il survient une complication imprévue, le chirurgien peut être amené à traiter cette complication sans réveiller le malade. L'acte chirurgical sous anesthésie implique donc une délégation d'autonomie du malade au médecin pendant la durée de l'anesthésie. Le chirurgien agit en respectant ce qu'il sait des volontés du malade mais en cas de complications imprévues, il tente, en application du principe de bienfaisance, de faire tout ce qu'il peut dans l'intérêt du malade. Enfin, même en l'absence d'évènements justifiant une modification de la stratégie chirurgicale, l'acte chirurgical sous anesthésie a cette particularité de ne pas permettre au malade d'exercer son autonomie pendant la chirurgie car il exclut tout retrait du consentement pendant le déroulement de l'acte chirurgical.

\section{La chirurgie d'exérèse en condition éveillée et le retrait du consentement perinterventionnel}

La chirurgie en condition éveillée permet au malade d'exercer son droit au retrait du consentement pendant toute la durée de son éveil: on peut donc considérer que, d'une certaine manière, il rejoint la condition habituelle des situations de soins ou de protocole de recherche clinique au cours desquels l'autonomie du malade persiste pendant la réalisation du soin ou du protocole et permet ainsi au malade de retirer à tout moment son consentement. Mais, de même qu'en cas d'expression du consentement se pose la question de la « compétence décisionnelle » du malade, doit-on de la même manière rechercher la compétence décisionnelle du sujet au moment où il retire son consentement?

L'évaluation de la compétence décisionnelle a été étudiée chez des malades présentant une maladie d'Alzheimer (14) ou chez des malades atteints de troubles psychiatriques comme la dépression ou la schizophrénie $(15,16)$. L'échelle d'évaluation de la compétence de MacArthur (17) permet d'analyser quatre capacités essentielles pour consentir à un traitement ou à une recherche: la compréhension du protocole, l'appréciation, le raisonnement et le choix. La compréhension concerne les autres alternatives, les bénéfices et les risques attendus. L'appréciation désigne la manière dont le malade met en perspective les bénéfices et les risques avec sa propre situation; le raisonnement désigne la capacité du malade à comparer les options thérapeutiques qui s'offrent à lui. Reste alors la capacité d'exprimer un choix et de le maintenir. Lors de l'éveil programmé au cours de l'acte chirurgical, il n'est pas possible de déployer les investigations neuropsychologiques nécessaires à l'évaluation approfondie des compétences décisionnelles. On peut seulement remarquer qu'au moment du recueil du consentement, notre malade n'avait pas de dysfonctionnement cognitif significatif; il avait quelques perturbations de ses compétences émotionnelles et une légère anxiété. Il n'avait pas de perturbation significative des fonctions exécutives et même son score en mémoire de travail s'était normalisé (Tableau 1). En outre il était éclairé sur les conditions de l'acte chirurgical: il avait été informé verbalement et surtout il avait déjà vécu une première intervention chirurgicale au cours de laquelle, lors de son réveil, il s'était déclaré trop fatigué pour réaliser les tests qui lui étaient proposés: l'intervention avait alors été interrompue et différée. Mais est-il légitime d'évaluer la 
capacité du malade à retirer son consentement ou doit-on considérer que le retrait d'un consentement est une manifestation exemplaire de l'autonomie et n'a pas besoin de fondements rationnels? Dans le cas publié de retrait de consentement lors d'une implantation d'électrodes de stimulation cérébrale profonde pour maladie de Parkinson, les auteurs signalent que le retrait du consentement annule tout le bénéfice de l'acte chirurgical en en maintenant les risques qui ne sont plus contrebalancés (10); en outre le choix du malade engage aussi la famille qui ne peut plus espérer de soulagement dans la prise en charge quotidienne du malade. Ces considérations ont paru suffisantes pour que le chirurgien tente de persuader le patient d'accepter la poursuite de l'acte chirurgical. Et c'est ainsi que l'acte chirurgical fut abandonné au nom du respect dû à l'autonomie du malade et même si la poursuite de l'acte chirurgical aurait été bénéfique pour le patient. Ceci est une application dans la logique de la philosophie des Lumières du primat du principe d'autonomie sur le principe de bienfaisance. Kant avait en effet invité les hommes à « oser savoir » et à « se servir de leur propre entendement », sans « la direction d'autrui » (18). Mais il $\mathrm{y}$ a lieu de distinguer une autonomie « de fait » et une autonomie « de droit ». En effet comme l'avaient souligné Beauchamp et Childress (19), certaines théories de l'autonomie considèrent qu'une personne autonome doit avoir les capacités de compréhension et de jugement nécessaires pour exercer un choix. Ceci est fidèle d'une certaine manière à la pensée de Kant car à défaut de se servir de son « propre entendement », la personne n'est pas réellement autonome mais hétéronome. Néanmoins la manifestation d'un retrait du consentement, même si le médecin estime que cette décision est contraire à l'intérêt du malade, constitue, en droit sinon de fait, une manifestation de l'autonomie, à laquelle on ne voit pas comment le médecin pourrait s'opposer. Il est par ailleurs difficile d'imaginer comment la personne de confiance, si elle a été désignée, ou la famille, pourrait choisir de contraindre un malade même si elles désapprouvent sa décision. C'est pourquoi d'ailleurs dans le cas de révocation du consentement au cours d'une chirurgie éveillée pour stimulation cérébrale profonde (10), l'équipe médicale, persuadée que la poursuite de l'intervention était le meilleur choix, tenta de convaincre le malade d'accepter la poursuite de l'implantation donc de revenir sur son retrait de consentement. Le refus du malade les résolut à abandonner la chirurgie. Dans notre cas de chirurgie éveillée pour exérèse optimale d'un gliome, le malade ayant eu en préopératoire toutes les informations nécessaires, il est apparu que l'essentiel était de s'assurer que le malade maintenait son choix. Il faut d'ailleurs noter que le malade ne tente aucune argumentation rationnelle. Il déclare une soif et une fatigue et ces deux sensations démobilisent toute sa motivation pour se soumettre aux tests peropératoires. Or en chirurgie éveillée il a auparavant été expliqué au malade qu'il sera « un acteur essentiel de l'intervention », à laquelle il doit participer activement. La fatigue rendait donc compte de l'incapacité que ressentait le malade à participer activement à l'intervention; il sollicitait même de s'en tenir à un rôle passif en réclamant d'être anesthésié.

\section{Comment interpréter ce retrait de consentement}

Soif et fatigue ont été les deux raisons invoqués par le malade pour retirer son consentement.

Dans le cas rapporté par Ford d'un retrait de consentement lors d'une intervention pour mise en place d'une stimulation cérébrale profonde, les auteurs envisagent le rôle favorisant de l'anxiété peropératoire dans le retrait de consentement au cours d'interventions neurochirurgicales par ailleurs longues. Ils préconisent d'ailleurs de porter une attention particulière au traitement de l'anxiété chez ces malades. Dans notre observation, le malade avait une légère élévation du score d'anxiété à l'échelle HAD lors du bilan neuropsychologique préopératoire. Mais il ne fut pas relevé de manifestations anxieuses pendant son réveil. La sensation de soif ou même de fatigue ne pouvaient-elles pas représenter des manifestations d'anxiété somatisée? Aucun argument n'a pu être recueilli en faveur de cette hypothèse. Quand il sera interrogé après son intervention, il ne se souviendra pas avoir ressenti d'anxiété particulière.

Peut-on évoquer une diminution de ses capacités d'insight? Cette hypothèse n'est guère probable même si l'insight n'a pas été exploré de manière spécifique. En tout cas en période préopératoire le patient avait correctement perçu qu'il devait être opéré d'une tumeur cérébrale. En outre, au moment de son retrait de consentement, le patient a demandé d'être à nouveau anesthésié, ce qui montre qu'il avait tout à fait conscience d'être en cours d'intervention chirurgicale.

Le malaise général entraîné par la soif et la fatigue a-t-il pu suffire à entraîner le retrait de consentement? Les perturbations cognitivo-émotionnelles même modestes constatées chez ce malade ont-elles pu l'empêcher de réagir de manière adaptée à sa sensation de soif et de fatigue et ainsi précipiter sa détermination à retirer son consentement? On sait aussi que les lésions de l'insula peuvent entraîner des difficultés de prise de décision en atténuant les capacités d'évaluation des risques (20). Une intervention persuasive aurait-elle pu être tentée dans le cadre d'un contrat contraignant de type « pacte d'Ulysse »?

Un contrat de type «pacte d'Ulysse » aurait-il pu être proposé pour tenter de ne pas enfermer le malade dans la « perte de chance » liée à son retrait de consentement?

Le pacte d'Ulysse (21) a été proposé en pathologie psychiatrique quand un malade psychotique, en rémission et jugé compétent, mais conscient par son expérience des rechutes antérieures des désordres causés à sa vie personnelle et familiale, sachant que lors de ces rechutes il ssopposera à tout traitement, demande « contractuellement » au psychiatre de lui administrer le traitement nécessaire même sous la contrainte au cas où une nouvelle rechute surviendrait. En effet au chapitre XII de l'Odyssée, Ulysse sur les conseils de la magicienne Circé, tout à la fois désireux d'écouter le chant des Sirènes mais d'échapper à la mort promise à ceux qui les approchaient, demanda à ses compagnons de se boucher les oreilles avec de la cire tandis qu'ils l'attacheraient solidement au mât du navire afin qu'il puisse « goûter le plaisir » d'entendre les chants sans risquer de le rejoindre. Et effectivement Ulysse raconte comment il fronça les sourcils pour donner à ses gens l'ordre de le défaire, mais eux, suivant ses instructions préalablement données, vinrent au contraire « resserrer ses liens ». C'est grâce au contrat préalable et à la contrainte qu'il permit à ses compagnons d'exercer sur lui qu'Ulysse eut la vie sauve (22). Doit-on considérer qu'en le maintenant attaché, les compagnons d'Ulysse attentèrent à son autonomie? Doit-on au contraire considérer que les compagnons d'Ulysse ont respecté son autonomie telle qu'elle s'exprimait quand, avant la rencontre des Sirènes, il pouvait réellement obéir à sa propre loi tandis qu'en écoutant les chant des Sirènes il s'exposait au contraire à être soumis à leur influence, donc à devenir hétéronome? On peut penser sur le plan éthique que les compagnons d'Ulysse ont 
effectivement respecté son autonomie réelle en protégeant Ulysse pendant le temps où il risquait de ne plus pouvoir exercer son autonomie en subissant l'influence hétéronomique du chant des Sirènes. Mais ne peut-on pas penser aussi qu'un sujet pourrait choisir d'abandonner son autonomie pour succomber au chant des Sirènes? On voit la difficulté qu'il peut y avoir à appliquer les catégories kantiennes aux situations pratiques. Et si le pacte d'Ulysse est transposé en pathologie mentale, on voit mal comment le contrat suffirait à soigner le malade sans faire intervenir le juge de manière variable selon les pays, dans le cadre d'une « hospitalisation sous contrainte ». Si les Etats (dont la France par la loi du 5 juillet 2011) encadrent les soins sous contrainte d'une procédure judiciaire complexe, c'est bien pour veiller à ne pas porter atteinte aux libertés fondamentales du malade dont l'intensité des troubles et les risques qu'ils font courir au malade et à son entourage justifient des soins non acceptés. En tout cas notre observation n'incite pas à mettre en œuvre pour les malades une procédure contractuelle de type pacte d'Ulysse. Quel que soit son aspect discutable sur le plan légal, les arguments éthiques ne manquent pas. Le malade, dont le bilan neuropsychologique avait montré une légère apathie, une légère anxiété et un déficit émotionnel n'avait pas été jugé incompétent lors du recueil du consentement. On a vu que les lésions insulaires peuvent diminuer les capacités d'évaluation des risques. D'une manière générale, le malade n'a pas à argumenter un retrait de consentement à des soins ou à un protocole de recherche et ce quelles que soient ses capacités cognitives. Le médecin doit faire preuve de qualités d'écoute et tout au plus lui expliquer pourquoi le consentement aux soins serait pout lui une source de mieux-être. Dans le cas particulier de la chirurgie éveillée, la mise en place d'un contrat de type pacte d'Ulysse ne peut en aucun cas redonner au malade le désir de participer activement aux épreuves neuropsychologiques qui lui sont proposées s'il n'en ressent, comme notre malade, ni la force ni l'envie. Néanmoins un contrat de type pacte d'Ulysse dans une forme atténuée pourrait permettre, lors du recueil de consentement, de préciser qu'en cas de retrait du consentement en cours d'interventionle chirurgien rappellera au malade qu'il avait antérieurement consenti à participer activement à l'acte chirurgical en étant réveillé. Le chirurgien lui rappellera aussi qu'il doit exécuter les tests proposés afin de bénéficier d'une exérèse optimale de sa lésion et que le maintien de son refus reviendrait à se contenter d'une chirurgie classique qui serait pour lui une diminution de ses chances d'amélioration, de rémission ou de guérison. Cette proposition mérite d'être débattue même si pour le cas rapporté dans ce travail et pour les autres cas opérés en condition de chirurgie éveillée le retrait de consentement n'a pas été anticipé au moment du recueil du consentement.

\section{Conclusions}

Le retrait de consentement est certainement rare en chirurgie tumorale en condition éveillée: dans notre expérience il s'agit du seul cas sur cent-soixante interventions. Compte-tenu des enjeux éthiques du retrait du consentement, la question se pose à savoir si cette éventualité ne devrait pas être abordée systématiquement parmi les informations données au sujet lors du recueil du consentement. Ceci consisterait au minimum à prévenir le malade en préopératoire que, lors de son réveil peropératoire, il pourra demander l'interruption de la procédure s'il la juge trop pénible. Cette proposition mériterait d'être débattue. L'information sur le retrait de consentement fait partie des informations données à tout sujet à qui est proposée l'inclusion dans un protocole de recherche clinique ou d'un prototerait d'être débattue. L'information sur le retrait de consentement fait partie des informations docole médical de soins. En chirurgie cette information n'est pas habituelle puisque l'anesthésie générale ne permet pas le retrait peropératoire du consentement, ce que permet la chirurgie en condition éveillée.

\section{RÉFÉRENCES}

1. Gil RT, Pluchon G, Micheneau C, et al. Une méthode d'évaluation rapide des fonctions cognitives (ERFC). Son application à la démence sénile de type Alzheimer. La Semaine des Hôpitaux de Paris. 1986;62(27):2127-33.

2. Slachevsky A, Villalpando J, Sarazin M, Hahn-Barma V, Pillon B, Dubois B. Frontal assessment battery and differential diagnosis of frontotemporal dementia and Alzheimer disease. Arch Neurol. 2004;61:1104-7.

3. Lezak MD HD, Loring DW. Neuropsychological assessment. 4th ed. Oxford: Oxford University Press; 2004.

4. Sockeel P, Dujardin K, Devos D, Deneve C, Destee A, Defebvre L. The Lille apathy rating scale (LARS), a new instrument for detecting and quantifying apathy: validation in Parkinson's disease. J Neurol Neurosurg Psychiatry. 2006;77:579-84.

5. Snaith RP. The hospital anxiety and depression scale. Health and Quality of Life Outcomes. 2003;1(29):1-4.

6. Ekman P. Facial expressions. In: Dalgleish TDP, M.J., editor. Handbook of cognition and emotion. New York: John Wiley and Sons, Ltd; 1999. p. 301.

7. Ekman P. Basic emotions. In: Dalgleish TDP, M.J., editor. Handbook of cognition and emotion. New York: John Wiley and Sons, Ltd; 1999. p. 45.

8. Loas G, Fremaux D, Marchand MP, Chaperot C, Dardennes R. L'alexithymiechez le sujet sain: validation de l'échelle d'alexithymie de Toronto (TAS) dans une population tout venant de 144 sujets, application au calcul de la prévalence. Annales medicopsychologiques. 1993;151(9):660-3.

9. Kleihues PC, W. K. Tumours of the nervous system - pathology and genetics. Lyon: International Agency for Research on Cancer; 2000.

10. Ford PJ, Boulis NM, Montgomery EB Jr., Rezai AR. A patient revoking consent during awake craniotomy: an ethical challenge. Neuromodulation: journal of the International Neuromodulation Society. 2007;10(4):329-32.

11. Europe Co. Additional Protocol to the Convention on Human Rights and Biomedicine, concerning Biomedical Research. 2005 [cited; Available from: http://conventions.coe.int/Treaty/EN/Treaties/ $\mathrm{Html} / 195 . \mathrm{htm}$

12. UNESCO. Cinquième Session du Comité Intergouvernemental de Bioéthique. 2007 [cited; Available from: http://unesdoc.unesco. org/images/0015/001512/151272f.pdf

13. AMM. Déclaration de Lisbonne de l'Association Médicale Mondiale sur les Droits du Patient. 2005 [cited; Available from: http://www. wma.net/fr/30publications/10policies/14/

14. Kim SY, Caine E, Currier GW, Leibovici A, Ryan JM. Assessing the competence of persons with Alzheimer's disease in providing informed consent for participation in research. Am J Psychiatry. 2001;158(5):712-7.

15. Appelbaum PS, Grisso T. The MacArthur Treatment Competence Study. I: Mental illness and competence to consent to treatment. Law and Human Behavior. 1995;19(2):105-26.

16. Carpenter WT Jr., Gold JM, Lahti AC, et al. Decisional capacity for informed consent in schizophrenia research. Arch General Psychiatry. 2000;57(6):533-8.

17. Grisso T, Appelbaum PS, Mulvey EP, Fletcher K. The MacArthur Treatment Competence Study. II: Measures of abilities related to competence to consent to treatment. Law and Human Behavior. 1995;19(2):127-48.

18. Kant E. Qu'est-ce que les lumières? Traduction Piobetta ed; 1784.

19. Beauchamp TLC, J. F. Les principes de l'éthique médicale. Paris: Les Belles Lettres; 2008. 
20. Clark L, Bechara A, Damasio H, Aitken MRF, Sahakian BJ, Robbins TW. Differential effects of insular and ventromedial prefrontal cortex lesions on risky decision-making. Brain. 2008;131(5): 1311-22.
21. Dresser R. Bound to treatment: the Ulysses contract. The Hastings Center report. 1984;14(3):13-6.

22. Homère. L'Odyssée, "poésie homérique". Paris: Les Belles Lettres; 1946. 